\title{
Note on Volume Effect in Coiling Molecules
}

\author{
By Robert Simha
}

\begin{abstract}
An estimate of the magnitude of the interference effect in a coil-like molecule is made by calculating the nearest neighbor density $w$ around a given link as a function of the total number of contributing chain units. It is shown for instance, that in a chain consisting of several hundred units, the first 15 contribute about 75 percent of the total nearest neighbor density at distances below the length of a link. The plots presented indicate that after about the first 20 links, the rate of increase of nearest neighbor density begins to flatten off. It is also possible to derive the modification of the distribution function of chain-ends in real chains for a given form of $w$.
\end{abstract}

The familiar model of a chain molecule currently used consists of an array of links of fixed length, making a fixed valence angle with each other, and able to rotate relatively to each other freely or in a manner restricted by potential barriers. This model is amenable to the treatment given by Rayleigh [1] ${ }^{1}$ in his theory of random flight. For a linear chain of $i$ links, it leads to the well-known result for the probability of finding a distance within an interval $r, r+d r$ between the two ends:

$$
p_{i}\left(\frac{r}{l}\right) d r=\left(\frac{3}{2 \pi l^{2}}\right)^{3 / 2} \frac{1}{i^{3} / 2} \exp \left(-\frac{3 r^{2}}{2 l^{2} i}\right) 4 \pi r^{2} d r
$$

In dealing with the model of a chain molecule of fixed valence angle, $l$ is equal to the length of a link corrected for the valence angle and for restricted rotation due to a potential barrier around each link, ${ }^{2}$ [2, 3]. Equation 1 holds strictly only in the limit $i \rightarrow \infty$. Using exact equations for small values of $i$ [1], one finds, e. g., for $i=6, p_{6}\left(\frac{1}{4}\right)=0.017$, according to eq 1 , as compared with the exact value of 0.015 . As the correction for restricted rotation amounts to changing the effective magnitude of $l$, it is possible to replace the actual chain by an effective one possessing free rotation but consisting of $i_{\text {eff }}<i$ "segments" of length $l_{\text {eff }}>l[4]$.

From eq 1 we can derive the aggregate probability ${ }_{\nu}^{N} \mid f_{c}(r) 4 \pi r^{2} d r$ of finding the endpoint of any link $i, \nu \leqq i \leqq N$, at distances between $r$ and $r+d r$ from one end of the chain, vz.

$$
{ }_{\nu}^{N} \mid f_{c}(r) 4 \pi r^{2} d r=\sum_{i=\nu}^{N} p_{i}\left(\frac{r}{l}\right) d r \doteq
$$

\footnotetext{
${ }_{1}^{1}$ Figures in brackets indicate the literature references at the end of this paper.

${ }^{2} \mathrm{P}$. Debye, lectures presented on various occasions.
}

$$
6 \frac{r}{l}\left[\Phi\left(\sqrt{\frac{3}{2 \nu}} \frac{r}{l}\right)-\Phi\left(\sqrt{\frac{3}{2 N} \frac{r}{l}}\right)\right] \frac{d r}{l} .
$$

Here the sum has been replaced by an integral. The function $\Phi$ is the error integral:

$$
\Phi(\xi)=\frac{2}{\sqrt{\pi}} \int_{0}^{\xi} e^{-x 2} d x
$$

In these considerations, configurations that allow different parts of the chain to occupy the same volume element are not excluded; in other words, volume effects are not taken into account. It is the purpose of this note to present essentially on the basis of results in eq 1 and 2 that constitute a zero approximation in respect to the above effect, a rough estimate of the magnitudes of the effects involved and particularly to examine the extent of the relative contributions of the links farther apart from a reference link to the volume effect. Let us now consider the probability $w(r) 4 \pi r^{2} d r$ of finding a nearest neighbor within a spherical shell with its center at one end of the chain. For values of $r$ smaller than $l$, this function will be considered as a relative measure for the occurrence of interference, although such distances can also be realized without interference. In the limit of $r \rightarrow 0$, the function $w$ becomes, of course, identical with $f$. For finite distances, $w$ can be obtained by a slight extension of an argument first given by Hertz [5]. Let $f(r) d \tau$ represent the number of points in a volume element $d \tau$, within a spherical shell between $r_{2}$ and $r_{1}$, such that

$$
\int_{r_{1}}^{r_{2}} f(\rho) d \tau=N
$$


where $N$ is the total number of points. Then the following relation exists between $w$ and $f$ :

$\left[1-\int_{r_{1}}^{r} w(\rho) 4 \pi \rho^{2} d \rho\right] \cdot \frac{N f(r) 4 \pi r^{2} d r}{\int_{r}^{r_{2}} f(\rho) 4 \pi \rho^{2} d \rho}=w(r) 4 \pi r^{2} d r$.

The validity of this equation can be seen readily. The probability of finding a nearest neighbor between $r$ and $r+d r$ (the right-hand side of eq 3 ) equals the probability of finding no nearest neighbor at a distance smaller than $r$ (the factor in the bracket) multiplied by the probability of one of the $N$ points being confined to a shell between $r$ and $r+d r$, if the space between $r_{1}$ and $r$ is empty (the second factor). Taking into account the normalization condition, eq 3 can be transformed by suitable differentiation into:

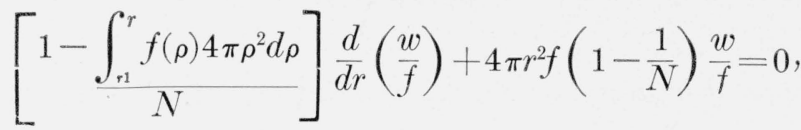

which has the solution:

$W=f\left[1-\frac{\int_{r_{1}}^{r} 4 \pi \rho^{2} f(\rho) d \rho}{N}\right]^{N-1} \doteq f \exp \left[-\int_{r_{1}}^{r} f(\rho) 4 \pi \rho^{2} d \rho\right]$.
In interpreting this result one notes that the factor $f$ on the left indicates the probability of finding a point in the volume element in question, and the quantity in the first bracket represents the probability of no point being in the volume between $r_{1}$ and $r$. This consideration could have been used directly to arrive at the expression for $w$. The second equation holds in the limit when $r$ is close to $r_{1}$ and $N$ is large.

In applying eq 4 to the problem under consideration, the distribution ${ }_{\nu}^{N} \mid f_{c}$ is inserted for the function $f$. For values of $\nu$ larger than or equal to 6 , eq 2 will be used. For values of $\nu$ between 2 and 6, Rayleigh's [1] exact polynomial expressions will be employed. For this purpose the function $p_{5}\left(\frac{r}{l}\right)$ not given in reference [1] was computed. The details of the calculation will be omitted. Setting: $x=\frac{r}{l}$ and $r_{1}$ in lf 4 equal to zero one finds

${ }_{2}^{5} f_{c}(r) 4 \pi r^{2} d r=\frac{x}{2}\left(1+\frac{21}{8} x-\frac{3}{8} x^{2}-\frac{1}{8} x^{3}\right) d x ; x<1$.

The integrations required by eq 4 , using eq 2 and $2 \mathrm{a}$ and setting $r_{1}$ equal to zero can be readily performed. Integration of eq 2, for instance, yields:

$$
\begin{aligned}
& \stackrel{\nu}{\nu} \mid \int_{j}^{r} f(\rho) 4 \pi \rho^{2} d \rho=2\left\{\frac{3}{2} x^{2}\left[\Phi\left(\sqrt{\frac{3}{2 \nu} x}\right)-\Phi\left(\sqrt{\frac{3}{2 N}} x\right)\right]-\frac{\nu}{2} \Phi\left(\sqrt{\frac{3}{2 \nu}} x\right)+\frac{N}{2} \Phi\left(\sqrt{\frac{3}{2 N}} x\right)+\sqrt{\frac{3 \nu}{2 \pi} x e^{-\frac{3}{2 \nu} x^{2}}--}\right. \\
& \sqrt{\frac{3 N}{2 \pi} x e^{\left.-\frac{3 x^{2}}{2 N}\right\}}}
\end{aligned}
$$

Figure 1 shows the contributions of links from the second to the $N$ th, inclusive, to the nearest neighbor probability $w(r) 4 \pi r^{2} d r$ as function of $N$ for values of $r / l=\frac{1}{4}$ and $\frac{1}{2}$, respectively, calculated from the pertinent equations. It can be seen for instance that the links from the fifteenth to the two hundredth relative to a reference link still make a contribution of the order of 25 percent to the total effect, although the larger part is of course due to the nearest links. Analogous conclusions may be drawn regarding interference around links in the interior of the chain or for shorter chains. In a chain with $N=50$, for instance, the first 15 members contribute 83 percent of the total nearest neighbor effect. As shown, the use of eq 1 for a short chain of six links is really not adequate. However, this equation makes the contribution for low $N$ 's larger and 


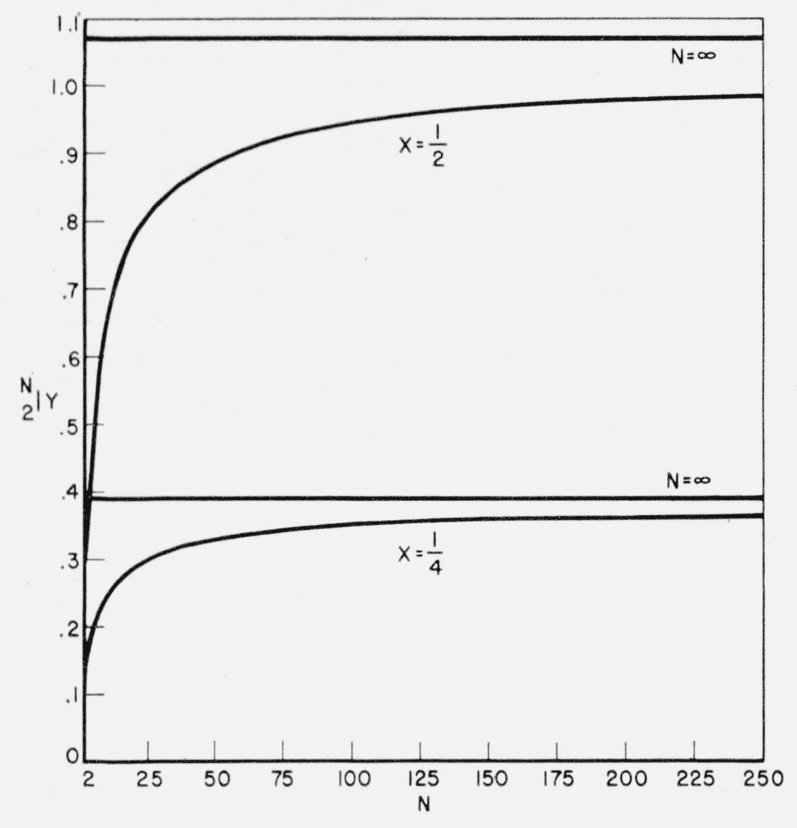

FIGURE 1. Contribution to the nearest neighbor density ${ }_{2}^{N} \mid w(r) 4 \pi r^{2}$, designated by ${ }_{2}^{N} \mid Y$, around a reference point as function of the number of successive links for $r=1 / 4$ and $r=7 / 2$. therefore does not alter the conclusion that the volume effect does not entirely originate from a small number of neighboring units in the chain. The segment size in "effective" chains referred to above would have to be quite large. The correct form of $w(r)$ would tend rapidly to zero on approaching a critical distance $r$ below $l$, and would assume the form used above for larger distances. Therefrom modified expressions for ${ }_{\nu}^{N} \mid f_{c}(r)$ and the distribution functious $p_{i}$ can be derived by utilizing the method discussed.

Mary Budge aided in the numerical calculations.

[1] Lord Rayleigh, Sci. Papers 6 (Cambridge Univ. Press, Cambridge, 1920); S. Chandrasekhar, Rev. Mod. Phys. 15, 1 (1943).

[2] C. Sadron, J. chim. phys. 43, 12 (1946).

[3] W. J. Taylor, J. Chem. Phys. 15, 412 (1947).

[4] W. Kuhn, Kolloid-Z. 68, 1 (1934).

[5] P. Hertz, Math. Ann. 67, 387 (1909); G. Jaffé, Phys. Rev. 58, 968 (1940).

Washington, August 12, 1947. 